\title{
Neuronavigation in a developing country: A pilot study of efficacy and limitations in intracranial surgery
}

\author{
S. Dwarakanath, A. Suri, B. S. Sharma, A. K. Mahapatra \\ Department of Neurosurgery, Neurosciences Center, All India Institute of Medical Sciences, New Delhi, India
}

\begin{abstract}
Context: Neuronavigation provides a patient-specific, three-dimensional (3-D) anatomy for preoperative planning and intraoperative navigation. However, the initial and maintenance costs are quite prohibitive, especially in the Indian scenario. Aims: To study the efficacy and limitations of neuronavigation, especially in the Indian scenario. Settings and Design: A prospective nonrandomized study. Materials and Methods: A total of 121 patients underwent intracranial surgery from 2002-2006, in which neuronavigation was used. In this, the initial part, we studied the efficacy and limitations of neuronavigation in the initial 37 patients. The efficacy of the image guidance was graded according to a point's scale in which points were awarded ranging from 0 to 3 . Cranial image guided score (IGS) was calculated by the summation of grading during designing the flap/burr hole, delineation of the intraoperative anatomy, navigation and access to the lesion and resection / biopsy of the lesion or completion of the procedure. The scoring ranged from 0-12 and the utility of IGS in cranial neurosurgical procedures was calculated based on the total points for each surgery. Results and Conclusion: Cranial image guidance was useful in a variety of operative steps. Intraoperative approach and navigation was relatively easier with an increase in perception of safety. Limitations of IGS include learning curve, cost and the phenomenon of brain shift. Drawbacks of the study included that this was a subjective rather than a truly objective study and the relatively lesser number of patients. We hope to conduct a larger study with randomization but the question of ethical approval would be a primary concern.
\end{abstract}

Key words: Cranial, grading system, image-guided surgery, neuronavigation

Image-guided neurosurgery (neuronavigation) or frameless stereotactic surgery, has made a tremendous impact over the past few years. It provides a patient- specific, three- dimensional (3-D) anatomy for preoperative planning and intraoperative navigation thus helping the surgeon to perform complicated procedures with improved accuracy and safety. ${ }^{[1,2]}$ Neuronavigation adds an entirely new dimension to neurosurgery. However, the initial capital expenditure makes the purchase quite prohibitive especially in developing countries like India. The question therefore arises whether the scarce public resources used for advanced systems can be justified or should they be used for other neurosurgical equipment. Another pertinent question is whether their usage adds significant value to the surgery.

In order to answer these questions, we decided on a prospective study to evaluate the efficacy and applicability of neuronavigation, especially in the scenario of a developing country.

\section{Aims and objectives}

1. To study the clinical profile, investigations, treatment, complications and outcome of patients undergoing surgery using neuronavigation.

2. To study the efficacy and limitations of neuronavigation in the surgery of intracranial lesions/ tumors.

\section{Materials and Methods}

The prospective study included all patients admitted to our hospital, who underwent surgery (cranial) under image guidance. A total of 121 patients have undergone surgery from 2002-2006 in which neuronavigation has been used. In the initial part of this study we used neuronavigation in a variety of cases in order to gauge the efficacy, intraoperative usage and limitations of the neuronavigation system. Thirty-seven consecutive patients who underwent image-guided cranial neurosurgical procedures at our department from March 2002 to July 2003 as a part of this pilot study were included (initial 37 patients of the 121 patients who underwent image- 
guided cranial neurosurgical procedures). The imageguided system i.e., Stealth station treatment guidance platform [Treon, Sofamor-Danek, Medtronic Inc, Memphis, TN, USA] was used.

Preoperatively, the patient's head was completely shaved and a set of 10 fiducials was applied and the imaging (CT/MRI) performed according to the specified protocol. The images were transferred to the workstation via the PACS and a 3-D image reformatted along with images in the sagittal, coronal and axial planes. Preoperative planning or virtual surgery was performed. Navigational or trajectory view and orthogonal or probe eye view were obtained. After induction, the patient's head was positioned using a 3 (three)- pin using either the Mayfield or the Sugita head clamp. The dynamic reference arc was attached and using the reference probe, fiducials were registered using the paired point registration. Imagepatient registration fusion was obtained. Once the patient was registered with the 3-D image at a specified accuracy level the surgery was proceeded with. Otherwise further surface-to-surface registration (surface merge) was performed to increase the accuracy. The incision site was marked and intraoperative position was checked periodically using reference probe whenever necessary.

The demographic characters of the patients were studied. The type of the lesion, extent of resection, duration of surgery and specific utility of neuronavigation were noted. Intraoperative course and problems encountered were noted. Postoperative course and complications were specified. Postoperative scans were performed when required to confirm the extent of resection and accuracy of biopsy/ shunt placement.

Grading utility of image-guided system (IGS) [Table 1]: A system for grading the efficacy of IGS was devised [Table 1a]. Cranial IGS score was calculated by the summation of grading during designing the flap/burr hole, delineation of the intraoperative anatomy, navigation and access to the lesion and resection / biopsy of the lesion or completion of the procedure [Table 1b]. The scoring ranged from 0-12 and the utility of IGS in cranial neurosurgical procedures was calculated based on the total points for each surgery.

Table 1: Image-guided surgery: Grading and utility score

\footnotetext{
1a: Grading scale of utility of image-guided system

0 : IGS not helpful or can be detrimental

$1+$ : IGS helpful but procedure can proceed without it

$2+$ : IGS more useful than conventional methods

$3+$ : IGS irreplaceable

1b: Cranial IGS score

(a) Design of flap/burr hole + b) delineation of anatomy $+c$ ) navigation/access to lesion + d) resection/ biopsy/procedure] 0-3: IGS not helpful or can be detrimental

4-6: IGS helpful but procedure can be continued without it

7-9: IGS more useful than conventional methods

10-12: IGS irreplaceable
}

IGS - Image-guided surgery

\section{Results}

Thirty-seven consecutive patients who underwent image-guided cranial neurosurgical procedures at the department of Neurosurgery, AIIMS, New Delhi, from March 2002 to July 2003 as a part of this pilot study were included. There were 25 males and 12 females in the study; their ages ranged from 7-68 years with a mean age of 36.5 years. The maximum number of patients were in their fourth (30.6\%) and fifth decades (20.4\%). There were 37 cranial surgeries conducted using the IGS [Table 2].

\section{Cranial image-guided surgery}

The most common cranial surgery using the IGS was for meningiomas (10/37) followed by gliomas (9/37). The details are summarized in Tables 2 and 3.

Regarding the positioning of craniotomy flaps/burr holes, IGS was invaluable $(3+)$ in designing and placement of burr holes for biopsies (thalamic glioma and metastasis) and shunt placement (loculated III ventricle) and small craniotomies for posterior frontal and parietal cavernomas. It was superior to conventional surgery $(2+)$ in placement of craniotomy for lesionectomy in intractable epilepsy, cavernous sinus hemangioma, posterior frontoparietal glioma, falcine orbital and intraventricular meningiomas, suprasellar epidermoid and cerebrospinal fluid

Table 2: Cranial image-guided surgery: Spectrum and indications $(\mathrm{N}=337)$

\begin{tabular}{lc}
\hline Indication & Nu m ber \\
Meningiomas & 10 \\
Clinoid/ medial sphenoid & 5 \\
Falcine & 2 \\
Foramen magnum & 1 \\
Intraventricular & 1 \\
Orbital & 1 \\
Gliomas & 9 \\
Frontal & 4 \\
Frontoparietal & 1 \\
Temporal & 1 \\
Midbrain & 1 \\
Thalamic (biopsy) & 2 \\
Cavernoma & 6 \\
Pons & 1 \\
Midbrain & 2 \\
Posterior frontal & 1 \\
Parietal & 1 \\
Cavernous sinus hemangioma & 1 \\
Epilepsy surgery & 3 \\
Functional hemispherotomy & 1 \\
Lesionectomy & 2 \\
Epidermoid & 1 \\
Interpeduncular & 1 \\
Suprasellar & 1 \\
Neurofibroma (Jugular foramen) & 1 \\
Chordoma (clivus) & 1 \\
Pituitary adenoma (recurrent) & 1 \\
Fibrous dysplasia & 1 \\
Cerebrospinal fluid rhinorrhea (recurrent) & 1 \\
Shunt placement (loculated third ventricle) & Parietal metastasis (biopsy) \\
\hline & 1 \\
\hline
\end{tabular}


Table 3: Cranial image-guided surgery: Clinico-radiological follow-up

\begin{tabular}{|c|c|c|c|}
\hline Indication & Intraop Impression & Clinical F/U & Radiological F/U \\
\hline \multicolumn{4}{|l|}{ Meningioma } \\
\hline Clinoidal (5) & Gross total & Excellent & No residual tumor $(3 / 5), \mathrm{NA}^{*}(2 / 5)$ \\
\hline Falcine (2) & Gross total & Excellent & No residual tumor $(1 / 2), N^{*}(1 / 2)$ \\
\hline F. Magnum (1) & Near total & Same & $N A^{*}$ \\
\hline Intraventricular (1) & Gross total & Excellent & No residual tumor \\
\hline Orbital (1) & Subtotal & Gamma knife (Vision PL -) & Small residual tumor \\
\hline \multicolumn{4}{|l|}{ Glioma } \\
\hline Frontal (4) & Gross total & Good [Astro $\|^{\prime}(2)$, III (1), IV (1)] RT" \pm CT" & No residual tumor (2/4)NA* (2/4) \\
\hline Temporal (1) & Subtotal & Good [Astro $I^{I I}$ RT\| & $\mathrm{NA}^{*}$ \\
\hline Fronto-parietal (1) & Subtotal & Good [Astro $\left.\|^{\prime I I}\right] \mathrm{RT}^{\|} \pm \mathrm{CT} T^{\natural}$ & $N A^{*}$ \\
\hline Midbrain (1) & Subtotal & Good [Oligo§] RT" & $N A^{*}$ \\
\hline Thalamic biopsy (2) & Biopsy & Good [Astro $\neq$ III (1), IV (1)] RT" \pm CTף & Biopsy \\
\hline \multicolumn{4}{|l|}{ Cavernomas } \\
\hline Pons (1) & Gross total & Expired & $N A^{*}$ \\
\hline Midbrain (2) & Gross total & 1-Poor, 1-Good & $N A^{*}$ \\
\hline Posterior frontal (1) & Gross total & Excellent & No residual lesion \\
\hline Parietal (1) & Gross total & Excellent & $N A^{*}$ \\
\hline Cavernous sinus (1) & Gross total & Excellent & No residual lesion \\
\hline \multicolumn{4}{|l|}{ Epilepsy surgery (3) } \\
\hline Hemispherotomy (1) & $\mathrm{na}^{\dagger}$ & No fresh seizures & \\
\hline Lesionectomy (2) & Gross total & $\begin{array}{l}\text { 1- no fresh seizures } \\
1 \text {-reduction in frequen }\end{array}$ & $\mathrm{na}^{\dagger}$ \\
\hline \multicolumn{4}{|l|}{ Epidermoid (2) } \\
\hline Interpeduncular (1) & Gross total & Excellent & Small residual tumor \\
\hline $\begin{array}{l}\text { Suprasellar (1) } \\
\text { indication }\end{array}$ & $\begin{array}{l}\text { Gross total Intraoperative } \\
\text { Impression }\end{array}$ & Excellent clinic & $\mathrm{NA}^{*}$ radiological $\mathrm{F} / \mathrm{U}$ \\
\hline Neurofibroma (1) & Near total & $\begin{array}{l}\text { Good, translal } \\
\text { Gamma knife }\end{array}$ & Residual tumor \\
\hline Chordoma (1) & Gross total & Excellent & $N A^{*}$ \\
\hline RecPit adenoma (1) & Subtotal & Excellent & $\mathrm{NA}^{*}$ \\
\hline Fibrous dysplasia (1) & Gross total & Lost to F/U & $N A^{*}$ \\
\hline Metastasis (Parietal) (1) & Biopsy & RT (lost to F/U) & Biopsy \\
\hline CSF rhinorrhea $(\operatorname{Rec})(1)$ & Closure & Excellent, no leak & $\mathrm{na}^{\dagger}$ \\
\hline Shunt placement (1) & Placement & Excellent & In situ, decompressed ventricle \\
\hline
\end{tabular}

*NA: Not available (postoperative imaging), †na: Not applicable, ${ }^{\ddagger}$ Astro: Astrocytoma, ${ }^{\circledR}$ Oligo: Oligodendroglioma, "RT: Radiotherapy, " $\mathrm{CT}$ : Chemotherapy

rhinorrhea. It was not of much use or equal to conventional surgery $(1+)$ in the placement of craniotomy flaps for skull base tumors, posterior fossa lesions, frontal and temporal gliomas, hemispherotomy, interpeduncular epidermoid and fibrous dysplasia [Table 4].

The IGS was invaluable in delineating the anatomy and providing access $(3+)$ to thalamic tumors, metastasis and loculated III ventricle, while it was more helpful than conventional surgery $(2+)$ in the rest except in hemispherotomy and epidermoid excision $(1+)$. The IGS was invaluable in determining the end point of resection/ biopsy $(3+)$ in thalamic glioma, brainstem glioma, clival chordoma and placement of the shunt while it was graded superior to conventional methods $(2+)$ in meningiomas (except orbital meningioma), posterior fronto-parietal glioma, brainstem cavernomas, lesionectomy for intractable epilepsy, planned subtotal excision of jugular foramen neurofibroma and recurrent pituitary adenoma. It was not very useful for determining the extent of resection or accuracy of procedure in epidermoids, functional hemispherotomy, frontal and temporal gliomas and CSF rhinorrhea and wherever there occurred a significant brain shift [Table 4].
Therefore, IGS was irreplaceable in patients who underwent biopsies or placement of shunt in the loculated ventricle (IGS score 12), while in other operations it was definitely more advantageous than conventional methods [Table 4].

\section{Mortality and morbidity}

There was no mortality or morbidity related to the usage of the image-guided system.

\section{Clinico-radiological follow-up}

The outcome is summarized in Table 3.

\section{Discussion}

IGS allows intraoperative navigation and consists of four fundamental parts, ${ }^{[1]}$ a method for registering the image with physical space, an intraoperative localization device (ILD), computer video display of medical images and methods for real time intraoperative feedback. Spetzger et al summarized a ten-year-long experience and presented an error analysis of 108 failures (12.4\%) in a total of 874 image-guided cranial neurosurgical procedures. ${ }^{[3]}$ 
Table 4: Cranial image-guided surgery: Utility

\begin{tabular}{|c|c|c|c|c|c|}
\hline Indication & $\begin{array}{l}\text { Designing } \\
\text { flap/burr hole }\end{array}$ & $\begin{array}{l}\text { Delineation } \\
\text { anatomy }\end{array}$ & $\begin{array}{l}\text { Navigation/ } \\
\text { access }\end{array}$ & $\begin{array}{l}\text { Procedure/ } \\
\text { resection }\end{array}$ & $\begin{array}{c}\text { Cranial } \\
\text { Image-guided surgery } \\
\text { score }\end{array}$ \\
\hline \multicolumn{6}{|l|}{ Meningioma } \\
\hline Clinoidal & $1+$ & $2+$ & $2+$ & $2+$ & 7 \\
\hline Falcine & $2+$ & $2+$ & $2+$ & $2+$ & 8 \\
\hline F. Magnum & $1+$ & $2+$ & $2+$ & $2+$ & 7 \\
\hline Intraventricular & $2+$ & $2+$ & $2+$ & $2+$ & 8 \\
\hline Orbital & $2+$ & $1+$ & $2+$ & $1+$ & 6 \\
\hline \multicolumn{6}{|l|}{ Glioma } \\
\hline Frontal & $1+$ & $2+$ & $2+$ & $1+$ & 6 \\
\hline Temporal & $1+$ & $2+$ & $2+$ & $1+$ & 6 \\
\hline Fronto-parietal & $2+$ & $2+$ & $1+$ & $2+$ & 7 \\
\hline Brainstem & $1+$ & $2+$ & $2+$ & $3+$ & 8 \\
\hline Thalamic biopsy & $3+$ & $3+$ & $3+$ & $3+$ & 12 \\
\hline \multicolumn{6}{|l|}{ Cavernomas } \\
\hline Pons & $1+$ & $2+$ & $2+$ & $2+$ & 7 \\
\hline Midbrain & $1+$ & $2+$ & $2+$ & $2+C$ & 7 \\
\hline Posterior frontal & $3+$ & $2+$ & $2+$ & $1+$ & 8 \\
\hline Parietal & $3+$ & $2+$ & $2+$ & $1+$ & 8 \\
\hline Cavernous sinus & $2+$ & $2+$ & $2+$ & $1+$ & 7 \\
\hline \multicolumn{6}{|l|}{ Epilepsy surgery } \\
\hline Hemispherotomy & $1+$ & $1+$ & $2+$ & $1+$ & 5 \\
\hline Lesionectomy & $2+$ & $2+$ & $2+$ & $2+$ & 8 \\
\hline Interpeduncular & $1+$ & $1+$ & $2+$ & $1+$ & 5 \\
\hline Suprasellar & $2+$ & $1+$ & & $1+$ & 6 \\
\hline
\end{tabular}

\section{Limitations of the image guidance system}

There are a few limitations to the neuronavigation system; since it depends on optical signals from the probe to the tracking cameras, a direct visual path must exist between the two components. ${ }^{[4]}$ Image-guided accuracy decreases as the surgery progresses due to fluid and tissue shifts, especially in cases of large tumors. Dorward et al studied the effect of brain distortion in 48 image-guided operations dividing them into four groups. ${ }^{[5]}$ He found more accuracy in skull base tumors, superficial extraaxial lesions and nonglial intraaxial lesions.

\section{Applications of image guidance/neuronavigation}

Breeuwer et al in a study of 300 cases operated using neuronavigation reported that neuronavigation had benefits in appreciation of anatomy, surgical planning, localization of lesion, extent of tumor resection, safety of surgery, effect upon confidence and also decreased duration of the surgery ${ }^{[6]}$ Henderson et al in their study of 150 patients reported that patients who underwent image-guided surgery had a significantly shorter length and cost of hospital stay, length of ICU stay and significantly lower cost of post ICU stay. ${ }^{[7]}$

In our preliminary study of 37 cases, we found IGS to be helpful in planning craniotomy, especially in avoiding air sinuses, limiting size and providing better centering. Precise marking was very helpful in lesions of the eloquent cortex, tumors for biopsy and placing shunt in the loculated III ventricle. We did not find it very useful in planning incisions for infratentorial lesions and skull base tumors. The anatomy was well delineated in virtually all cases and IGS helped in the presurgical planning, assessing alternate approaches and choosing the optimal approach. Intraoperative approach and navigation was relatively easier with an increase in the perception of safety. This was especially useful in skull base tumors and brainstem lesions where the extent of vermian splitting was limited. The IGS helped us identify and avoid important vascular structures. In lesions of the eloquent cortex and the brainstem, the cortical incision could be placed on the most superficial part of the lesion thus limiting the damage to the normal cortex. The IGS helped in estimating the extent of resection, especially in some skull base tumors, falcine and intraventricular meningiomas. We found IGS to be invaluable in biopsies and placement of the shunt. However, IGS use was limited due to the time consumed, especially for the setup. Although towards the end of the study the time taken had decreased due to the increased familiarity with the usage.

\section{Meningiomas and skull base tumors}

We found that IGS was helpful in planning and limiting the size of craniotomies, avoiding the air sinuses, providing access to the tumors, avoiding important vessels and determining the extent of resection of the tumors. Similarly in the eight skull base neoplasms, we found that IGS was extremely helpful in providing access to the tumor, delineating the margins, identifying the vascular and osseus relations and estimating the extent of resection..$^{[8-11]}$ 


\section{Gliomas}

In this study, IGS helped in optimizing incision in the frontoparietal and the thalamic gliomas, while in the standard frontal and temporal craniotomies it was not of much help. Though it helped in the initial corticectomy, as resection proceeded, it had limitations in accurately estimating the extent of resection. It was invaluable in the approach towards the thalamic tumor and an intraoperative assessment of the position of the biopsy needle could be made. ${ }^{[12]}$

\section{Cavernomas}

The IGS helps immensely in the planning of the incision, approach, placement of the cortical incision and estimating the extent of excision thus allowing gross total excision. ${ }^{[13]}$

\section{Miscellaneous}

The IGS was helpful in extratemporal lesionectomy and in hemispherotomies where it allows visualization of the anatomical landmarks. ${ }^{[14]}$ The IGS was invaluable in placing shunt tips in isolated and loculated ventricles. ${ }^{[15]}$ Similarly, the IGS helps immensely in the biopsies as accurate localization, trajectory planning and intraoperative visualization is possible with image guidance.

There have been a few drawbacks in the study. At our center we operate around 3000-3200 cases per year of which 1400-1500 are elective cranial cases. In spite of this load neuronavigation was sparingly used. The probable causes included the limited theater time available. The registration was taking up relatively more time, especially in the early stages, compared to cases where neuronavigation was not used. This probably partially explains the relative paucity of cases. Other probable causes include lack of trained technicians, and limitation of usage to a single operating theater. Initially we had lots of technical glitches and the programming staff had to come from another city to set the system right. There was also a relative usage bias. The senior faculty used the system less frequently when compared to the junior colleagues. Also, they used to grade the efficacy of the system less than their junior colleagues which raises the pertinent question of experience.

The other drawback is that this was a subjective analysis based on preset criteria giving rise to the question of objectivity. We did perform an extensive literature review but were unable to come upon any grading scale and so designed our own scale.

The main argument against neuronavigation has been the brain shift; however, the inaccuracies are not limited to the brain shift alone. There were errors of registration too. We experienced intraoperative inaccuracies but the most troublesome was that due to brain shift.

The present study on the application of image-guided system in cranial neurosurgical procedures is the first of its kind in India. Cranial image guidance was useful in designing the craniotomy flap or burr-hole, delineation of anatomy, navigation and access and extent of resection or accuracy of biopsy/ procedure. The main disadvantage of neuronavigation has been the intraoperative brain shift that occurs during surgery, thus making the preoperative imaging inaccurate. This can be overcome by using intraoperative imaging thus providing real time information. The problems regarding the increased time of surgery and the initial problems regarding usage will come down as more and more cases are performed. The other problem that can arise, especially in a developing country like ours, where there is a scarcity of resources, is the cost of the equipment and the cost-benefit ratio.

In a government-funded setup like ours the capital expenditure is not charged onto the patient, but in a private set up the costs incurred on the purchase and maintenance will definitely be passed onto the patient. In India where the majority of patients are uninsured these costs will strain their resources further. To make neuronavigation truly effective an intraoperative imaging system is required which will further push up the costs. Therefore the question arises as to whether the neuronavigation system is the instrument of choice to buy especially when there is a lack of intraoperative navigation? The IGS does have its advantages like increased perception of safety, probably increases the amount of tumor resection (though this will need larger studies along with a control group), along with improved accuracy which may lead to lesser complications. However, the high capital expenditure, absence of intraoperative imaging, lack of trained dedicated supporting technical staff does make the IGS a strain on the resources. The main drawback of this study is the subjective analysis. Therefore a larger randomized study with more objective criteria would be necessary to provide definitive answers.

However, the benefits of neuronavigation, which can only increase with the usage of intraoperative imaging, should offset the costs. The image-guided system is an important tool in the armamentarium of the neurosurgeon and does not replace him/her. Thus in this paper we seek to establish the efficacy and applications of neuronavigation and will follow it up in the second part of this paper where we shall discuss the optimization of usage and discuss our complete experience with neuronavigation.

\section{References}

1. Golfinos JG, Fitzpatrick BC, Smith LR, Spetzler RF. Clinical use of a frameless stereotactic arm: Results of 325 cases. J Neurosurg 1995;83:197-205.

2. Tasker RR. Stereotactic surgery: Principles and techniques in neurosurgery. Wilkins HW, Rengachary SS, editors. $2^{\text {nd }}$ ed. McGrawHill: 1996. p. 4069-89.

3. Spetzger U, Hubbe U, Struffert T, Reinges MH, Krings T, Krombach GA, et al. Error analysis in cranial neuronavigation. Minim Invasive Neurosurg 2002;45:6-10 
4. Warnick EP, Bath JS. Image guided neurosurgery. In: Schmidek HH. Operative neurosurgical techniques, $4^{\text {th }}$ ed. WB Saunders: chap 46. p. 611-27.

5. Dorward NL, Alberti O, Velani B, Gerritsen FA, Harkness WF, Kitchen ND, et al. Postimaging brain distortion, magnitude, correlation and impact on neuronavigation. J Neurosurg 1998;88:656-62.

6. Breeuwer M, Wadley JP, de Bliek, Buurman J, Desmedt PA, Gieles $\mathrm{P}$, et al. The Easi project-improving the effectiveness and quality of image guided surgery. IEEE Tran Inf Techno Biomed $1998 ; 2: 156-8$.

7. Henderson JM, Eichholz KM, Bucholz RD. Decreased length of stay and hospital costs in patients undergoing image-guided craniotomies. J Neurosurg 1997;86:367.

8. Paleologos TS, Wadley JP, Kitchen ND, Thomas DG. Clinical utility and cost-effectiveness of interactive image-guided craniotomy: Clinical comparison between conventional and image-guided meningioma surgery. Neurosurgery 2000;47:40-8.

9. Barnett GH. Surgical management of convexity and falcine meningiomas using interactive image-guided surgery systems. Neurosurg Clin North Am 1996;7:279-84.

10. Schul C, Wassmann H, Skopp GB, Marinov M, Wolfer J, Schuierer
G, et al. Surgical management of intraosseous skull base tumors with aid of operating arm system. Comput Aided Surg 1998;3:312-9.

11. McDermott MW, Gutin PH. Image-guided surgery for skull base neoplasms using the ISG viewing wand. Anatomic and technical considerations. Neurosurg Clin North Am 1996;7:285-95.

12. Wirtz CR, Albert FK, Schwaderer M, Heuer C, Staubert A, Tronnier VM, et al. The benefit of neuronavigation for neurosurgery analyzed by its impact on glioblastoma surgery. Neurol Res 2000;22:354-60.

13. Zamorano L, Matter A, Saenz A, Portillo G, Diaz F. Interactive image-guided resection of cerebral cavernous malformations. Comput Aided Surg 1997;2:327-32.

14. Olivier A, Alonso-Vanegas, Comeau R, Peters TM. Image-guided surgery of epilepsy. Neurosurg Clin North Am 1996;7:229-43.

15. Wagner W, Gaab MR, Schroeder HW, Sehl U, Tschiltschke W. Experiences with cranial neuro-navigation in pediatric neurosurgery. Pediatr Neurosurg 1999;31:231-6.

Accepted on 20-03-2007

Source of Support: Nil, Conflict of Interest: None declared. 